cinoma for surgical treatment the physician must satisfy himself that the patient has sufficient pulmonary reserve and cardiac vascular reserve to withstand pneumonectomy should that be necessary. He must be certain there are no metastases. He must satisfy himself that the phrenic nerves, the sympathetic nerves, and the recurrent laryngeal nerves are not involved. He must, by examining the barium swallow, be certain that mediastinal glands are not involved by growth, and he must satisfy himself that the great veins in the thorax are not compressed or invaded. At the same time he must realize that even a pleural effusion or involvement of a phrenic nerve do not inevitably signify that the growth is irremovable. Surgical excision is often the best prospect of cure and no patient should be considered to be beyond surgical aid without most careful deliberation.

Furthermore exploratory thoracotomy is without serious consequence if the tumour is irremovable.

It has recently been suggested that angiopneumography (Weiss et al., 195I) is an essential preoperative investigation in order to determine prospects of surgical removal. While this may be true in some centres such studies elsewhere will continue to take second place to surgical exploration.

\section{BIBLIOGRAPHY}

ANDERSON, H. J., DAVIDSON, H. C., RICHARDSON, A. T. (1953). In the press.

ANDERSON, H. J., and PIERCE, W. J. (1953). In the press. BROOKS, W. D W. DAVIDSON, M. THOMAS, C., PRICE, ROBSON, K., and SMITHERS, D. W. (195I), Thorax, 6, I DAVIDSON, M. (195I), 'The Diagnosis and Treatment of Intrathoracic New Growths,' Oxford University Press.

DENNY-BROWN, D. (1948), ₹. Neurol. Neuromy and Psychiatry, II, 73 .

DUDGEON, L. S. (1934), f. Laryngol., 50, 752.

GLEDHILL, E. J., SPRIGGS, J. B., BINFORD, C. H. (1949), Amer. F. Clin. Path., 19, 235.

GOOD, C. A., MCDONALD, J. R., CLAGGETT, O. T., and GRIFFITH, E. R., Amer. F. Roent., 64, I.

HAROLD, J. T. (1952), Q.F.M., 45, $21,353$.

MACHALE, S. J. (1953), Thorax, 8, 164.

MENDLOWITZ, M. (1942), Medicine, 21, 269.

MINOR, G. R. (1950), F. Thorac. Surg., 20, 34.

NICHOLSON, H. T. (I949), Thorax, 4, 125.

OCHSNER, A., and DEBAKEY, M. (1942), f. Thorac. Surg., II, 257.

PANCOAST, H. K. (1932), Ұ.A.M.A., 99, 1391.

PRICE THOMAS, C. (1948), 'British Surgical Practice,' Vol. 5, p. 450.

RIENHOFF, W. F., JR. (1944), Ұ.A.M.A., 126, I 123.

WYBURN MASON, R. (1948), Lancet, i, 203.

\title{
UNUSUAL MANIFESTATIONS OF CARCINOMA OF THE LUNG
}

By Joseph SMart, M.D., F.R.C.P. The London Chest Hospital

The increasing frequency of carcinoma of the bronchus is a problem which is of concern to all, and the results of treatment at the present time are somewhat disappointing, the most successful treatment being early surgical excision. It is important, therefore, that the early diagnosis of the condition should be recognized, and while the common manifestations of the disease such as haemoptysis, cough and loss of weight are well appreciated, it is important to recognize the more unusual modes of onset in order that it may be detected as soon as possible, thereby giving the patient the best chance of recovery.

The various ways in which carcinoma of the lung may present can be most readily appreciated if the nature of the lesion is borne in mind. That is to say that symptoms arise from one of two sources, either the primary growth itself or from secondary deposits occurring in distant parts of the body when the primary itself is of insufficient size to cause symptoms. Those symptoms which arise from the growth itself usually occur as a result of direct spread into the surrounding structures, which may involve the bronchi, the nerves, the veins, the oseophagus and the pericardium and myocardium.

In this article I propose to deal with the less common manifestations of carcinoma of the bronchus in two groups, firstly, the unusual initial symptoms and, secondly, the unusual later manifestations due to peripheral secondary deposits.

\section{Unusual Initial Symptoms}

The early symptoms of a primary pulmonary neoplasm which may be overlooked are those where the pulmonary symptoms are absent or where they suggest an acute pulmonary infection. These less common symptoms can be easily understood by considering the involvement of the various structures in the mediastinum, as these are invaded by the growth. 
Pressure on bronchus. The usual effect of pressure on a bronchus is to cause collapse of the distal portion of the lung, but before the bronchus is completely occluded there is a partial obstruction and this may give rise to various symptoms. There may, on occasions, be an initial wheeze noticed by the patient and, on examination, rhonchi and sibili are heard on one side of the chest. These signs are those of partial obstruction and must not be mistaken for chronic bronchitis where partial obstruction occurs owing to bronchial spasm; the important point in the differential diagnosis is that in chronic bronchitis with spasm the lesion is invariably bilateral, but when the rhonchi and sibili are entirely unilateral or involving just part of one lung this is usually strong evidence of organic partial obstruction and bronchoscopy is indicated. At this stage there may be little to be seen radiologically. A further symptom which may be misleading, but which is directly due to a pulmonary neoplasm and which occurs with partial obstruction, is the development of what appears to be a straightforward lobar pneumonia with sudden onset, rigor, temperature, general malaise, cough and sputum, which responds initially to chemotherapy but fails to clear completely. An X-ray of the chest often shows evidence of partial collapse, suggesting the true nature of the disease. Lastly, partial obstruction of a bronchus, particularly if it is in the lower lobe, may give rise to a lung abscess (Fig. I), with a high swinging temperature, a high white blood count, cough and foul sputum. The X-ray will probably show a large abscess cavity with a fluid level, but again the failure of this to respond to antibiotics and posture should raise the question of pulmonary carcinoma. Indeed, in any case of lung abscess occurring in the carcinomatous age group this possibility should always be borne in mind. Bronchoscopy will show a partial obstruction unless the lesion is too peripherally situated, in which case sputum examination for neoplastic cells is of considerable value.

Involvement of nerves. The recurrent laryngeal nerve is the nerve which is most commonly damaged, causing hoarseness of the voice. Rarely the right nerve is involved due to secondary deposits in the lymph glands of the neck and this is stressed because the left recurrent laryngeal nerve is frequently involved owing to its anatomical position and the possibility of right laryngeal nerve involvement is often forgotten. It should also be stressed that when the nerve is first paralyzed there may be no other symptoms referable to the chest and no radiological evidence of abnormality in the lung fields, although this is uncommon. Occasionally the hoarseness improves at first due to the opposite vocal cord moving right

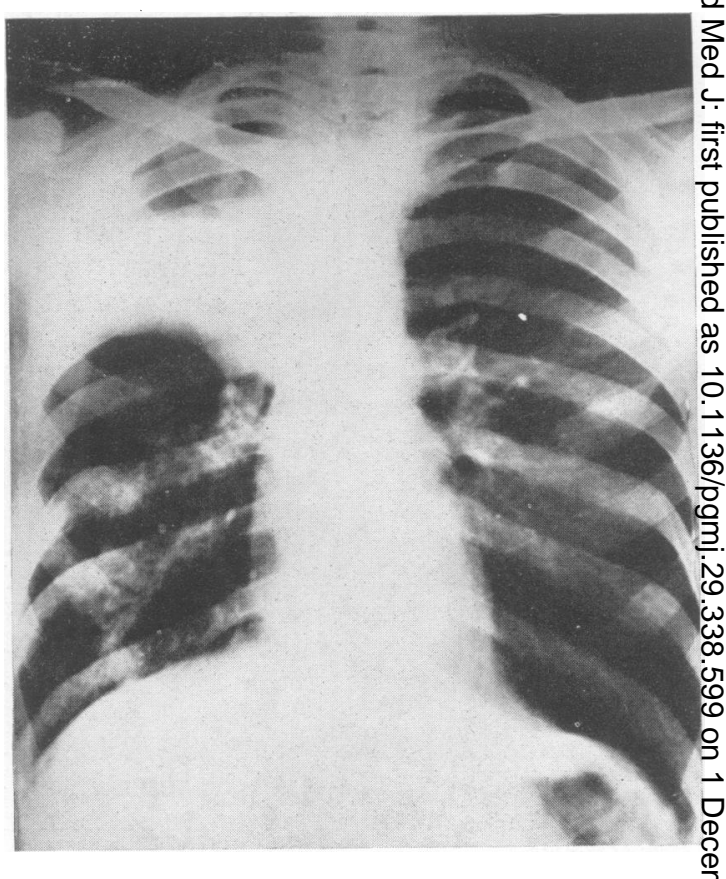

FIG. I.-Carcinoma of the bronchus, showing right apical cavity with fluid level.

across the larynx, which may suggest recovery the nerve lesion so that the true nature of disease is obscured. Another nerve involved is the phrenic nerve, giving rise to some slight dyspnoeas due to paralysis of the diaphragm and inadequate ventilation of the lungs. The sympathetic nervous system may also be affected where the ganglia ir? the upper part of the chest are destroyed $b \overrightarrow{\overrightarrow{5}}$ growth, giving rise to a Horner's syndrome. In the very early stages there may be stimulation of the sympathetic so that there is flushing on that side. of the face, widening of the palpable fissure an $\$$ dilatation of the pupil, but this stage often passes. unnoticed and the normal Horner's syndrome is the first thing that the patient complains of, that is pallor on that side of the face, lack of sweatinge ptosis of the upper lid and a small pupil.

Pressure on veins. The superior vena cava is the vein that is commonly involved because it has relatively long course in the mediastinum. The. inferior vena cava is largely outside the chest passing through the diaphragm directly into the right aurical, so this does not get obstructed. The symptoms in the early stages of superior vena caves obstruction may be quite vague, starting often wite a sense of fullness in the head and neck on bending or lying down owing to the venous back pressure caused by involvement of mediastinal glands by growth which cause pressure on, and may obstruct the superior vena cava. The slight enlargement of 


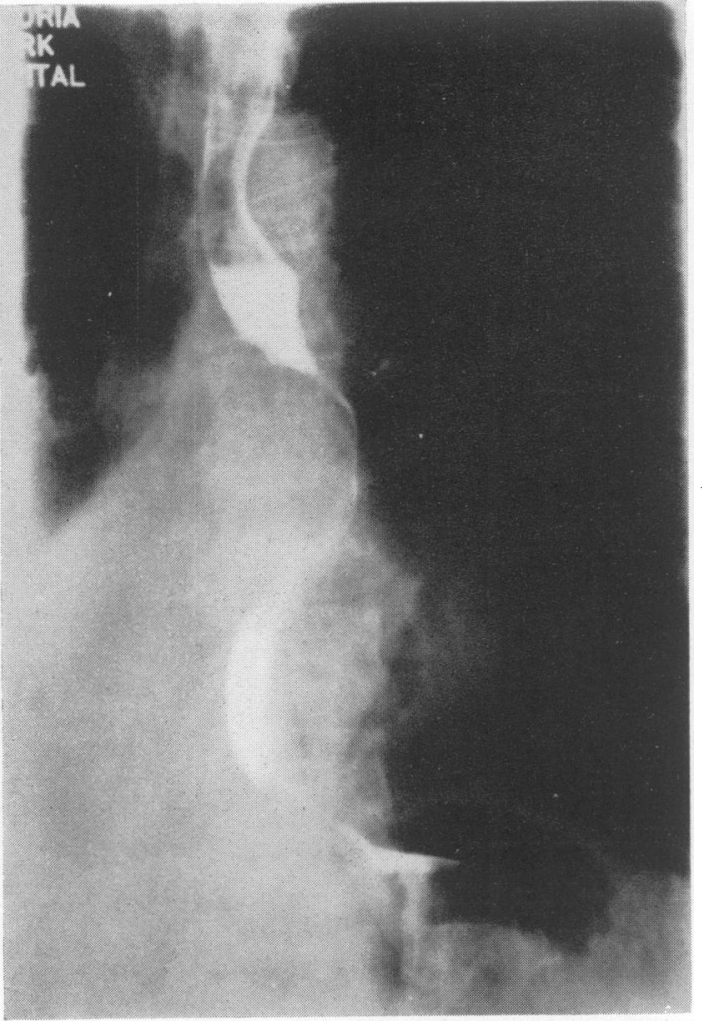

Fig. 2.-Barium swallow, showing obstruction of the oesophagus due to secondary glands in the mediastinum.

the neck at the onset is more commonly noticed by males than females as the former find their collars becoming tight. Later, when complete obstruction occurs, the patient develops gross oedema of the head, neck and arms together with the development of a colateral venous return on the chest wall. When the superior vena cava obstruction has become marked, the diagnosis is obvious, but in the early stages it may easily be missed. Very rarely obstruction of the veins of one arm may occur as the initial onset of symptoms, this being due to secondary deposits in the lymph glands above the clavicle pressing upon the brachial vein.

Pressure on oesophagus. Difficulty in swallowing due to pressure on the oesophagus from direct spread or secondary deposits in the mediastinal glands, which become enlarged and press upon the oesophagus, may occur. These symptoms may be mistaken for an oesophageal lesion, cardiospasm or even a functional lesion, but a barium swallow will demonstrate the fact that the oesophagus is being pressed upon from without (Fig. 2).

Involvement of the pericardium and myocardium. This is an unusual mode of onset but occasionally

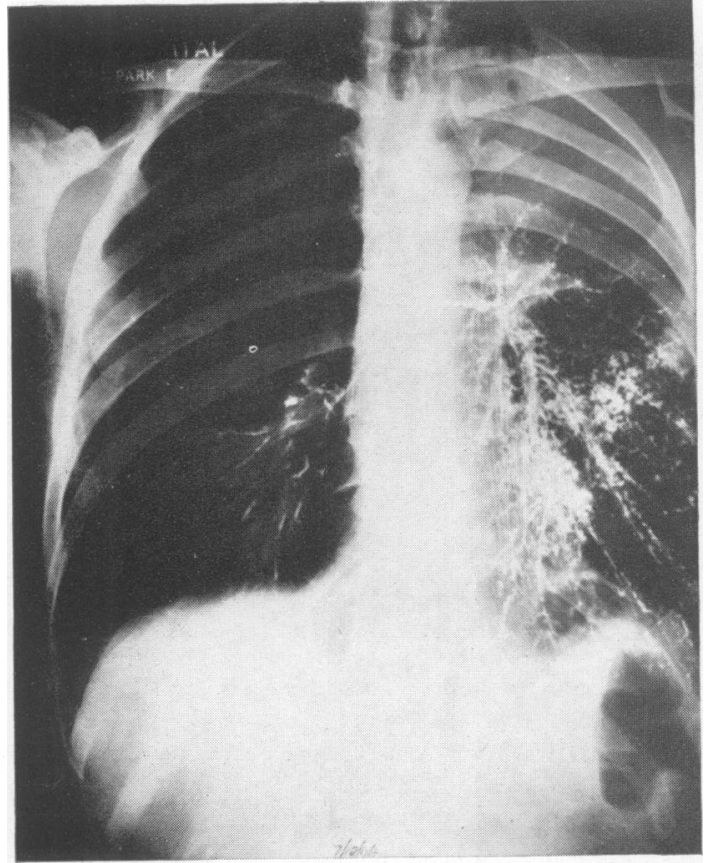

FIG. 3.-Superior sulcus tumour on left side, with $\bar{\Phi}$ lipiodol. Note-(I) Destroyed second rib from the clavicle anteriorly. (2) No displacement of the mediastinum.

cases occur. Cardiac irregularity and marked tachycardia are the initial symptoms. With the use of X-ray screening in cardiology, however, the true nature of the disease is apparent. It is remarkable that these symptoms are the initial symptoms for radiologically there is usually fairly gross pulmonary and mediastinal involvement. This is, as a rule, associated with the undifferentiated carcinomas. Another interesting point is that in carcinoma of the bronchus treated by deep X-rays, persistent tachycardia or a rising pulse rate, which had previously settled, is always suspicious of myocardial involvement.

All these modes of onset are primarily due to the growth itself either directly involving the surrounding structures, as in the mediastinum, or partially or completely blocking the bronchus or by direct spread to the pleura.

There are other modes of onset which should also be mentioned. The superior sulcus tumour (Fig. 3) which arises in the upper part of the lung in the periphery may grow to a considerable size without causing obstruction to the bronchus, and invade the ribs in the region of the second and third rib posteriorly, causing the initial symptom of pain in the shoulder going down the arm. Clinically there is another interesting point about 


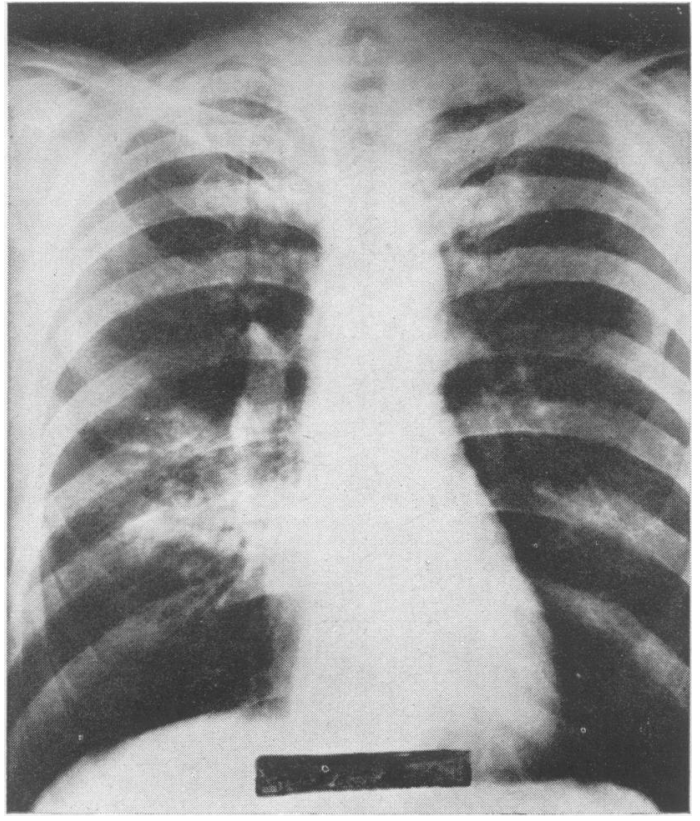

Fig. 4.-Carcinoma of the bronchus presenting as an intercurrent infection both clinically and radiologically.

these tumours and that is that the mediastinum tends to be pushed away from them by virtue of the size of the growth at the apex and not drawn towards the lesion, which is much more common, owing to collapse of a segment or lobe of the lung. An X-ray in such cases will show a homogenous mass with rib destruction, usually in the posterior part of the upper ribs.

The peripheral carcinoma also should be mentioned, because this may give rise to very few clinical abnormalities apart from such general symptoms as loss of weight, loss of appetite, slight anaemia, etc. Radiologically the lesion not infrequently looks like a low-grade infection, and the possibility of such a low-grade infection being carcinoma must be remembered (Fig. 4). Failure of such a lesion to clear with antibiotics would add to this suspicion. Bronchoscopy will not, as a rule, help, as only the major bronchi and their primary divisions can be seen, but sputum examination for neoplastic cells is extremely valuable.

A pleural effusion arising in a person of the carcinomatous age group may be the initial symptom of a growth. There may or may not be a pyrexial phase and there is usually no pain at the onset, but increasing dyspnoea causes the patient to seek advice. The cytology of the pleural fluid may help and plaques of neoplastic cells may be identified. These are more likely to be found in

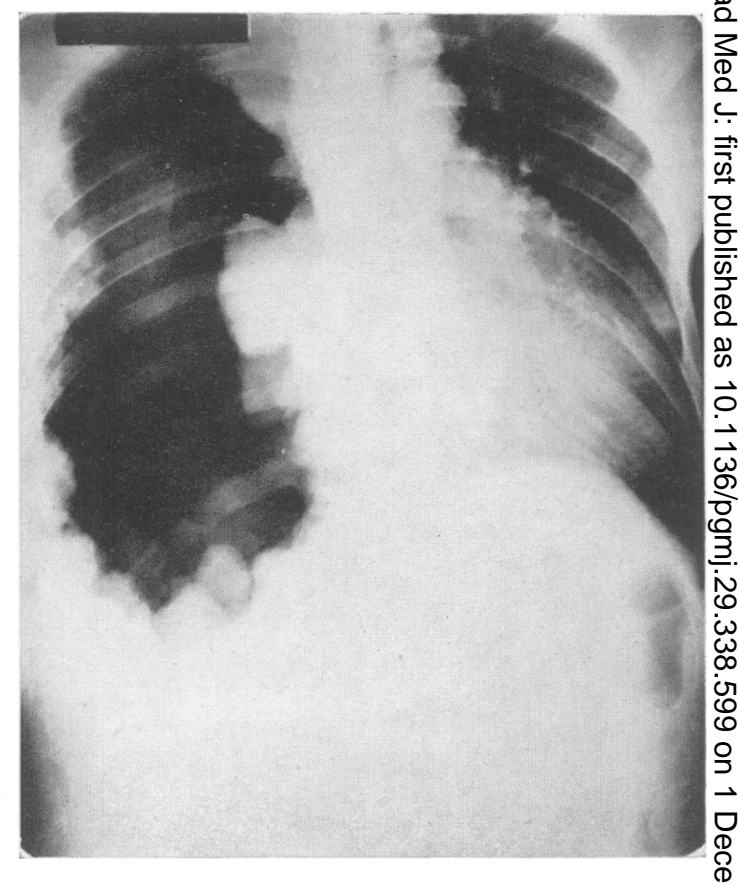

Fig. 5.-Mesothelioma of the pleura after removal of the fluid, showing pleural deposits.

the fluid if the exploring needle is inserted faiby low down in the chest posteriorly, especially 3 ? the patient has been lying in bed, as the cells tend? to gravitate to the base of the pleural cavity. Frequently the underlying lung lesion cannot be⿳亠丷厂 seen and there may be a collapsed lobe beneath the $\frac{\mathbb{Q}}{2}$ fluid. Normally, with collapse of a lobe of the lung, one would expect the mediastinum to be윽 displaced towards the lesion and conversely with a simple pleural effusion one would expect the mediastinum to be displaced away from the lesion, but when both occur together, as they may in 3 malignant disease of the lung, there is no displace-o ment. A pleural effusion which begins as clear?. coloured fluid and recurs rapidly, necessitating the removal of fluid which gradually becomes blood- -3 stained, is the usual onset of the so-called meso-o thelioma of the pleura (Fig. 5). Much discussion? has occurred with regard to this type of tumouro and there is still doubt whether it is primarily pleural in origin or whether it is due to a smallo peripheral carcinoma with marked secondary deposits in the pleural cavity.

Finally, a primary carcinoma or adenocarcinoma or sometimes a non-malignant growth such as a fibroma of the lung, may start with symptoms of polyarthritis, largely affecting the hands, but ites? may also affect the feet, with gross clubbing of the fingers and toes (Fig. 6a). This clubbing is much 


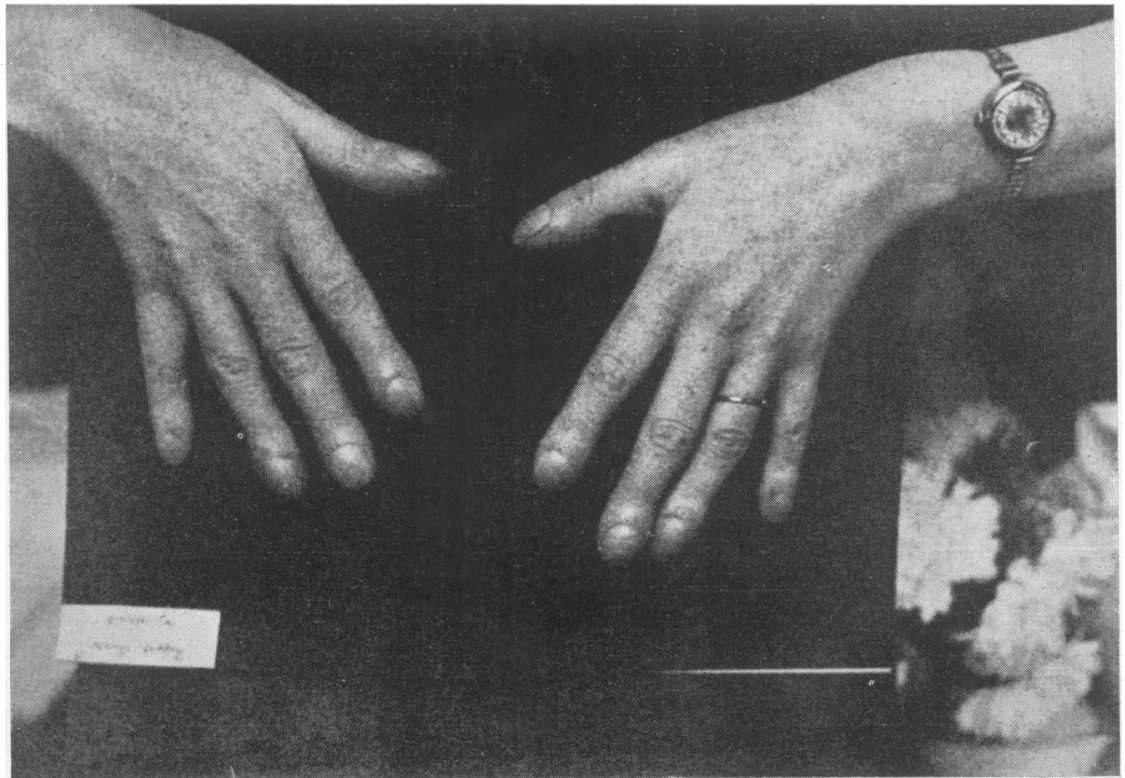

Fig. 6, a and b.-Adenocarcinoma of right lower lobe bronchus associated with gross clubbing of the fingers.

more marked than is usually seen with bronchiectatic patients and may be of quite an amazing degree, and develop very rapidly. At this time there are frequently no chest symptoms at all, but an X-ray of the chest will usually show a rounded shadow occurring in the lung fields (Fig. 6b). As these lesions are often peripherally situated, bronchoscopy may be negative, but it is interesting to note that when the lesion is removed surgically by lobectomy or pneumonectomy, the arthritis clears up very rapidly, being much improved even the next day, and the clubbing also rapidly diminishes. The reason for these arthritic changes and the clubbing has never yet been satisfactorily explained, nor is it clear why there should be such a rapid improvement as soon as the growth has been removed.

A further interesting and unusual mode of onset is the development of ataxia or lack of coordination, suggesting neurological disease, but this has been shown to occur with a primary pulmonary neoplasm, and is due to demeylination of the nerve fibres. Unlike the arthritis and clubbing there is no rapid change after pneumonectomy, but this may be due to the very slow rate of recovery of the nerves.

\section{Unusual Later Manifestations}

Unusual symptoms arising later in carcinoma of the bronchus are, as a rule, due to secondary deposits, therefore they may be manifold, and the

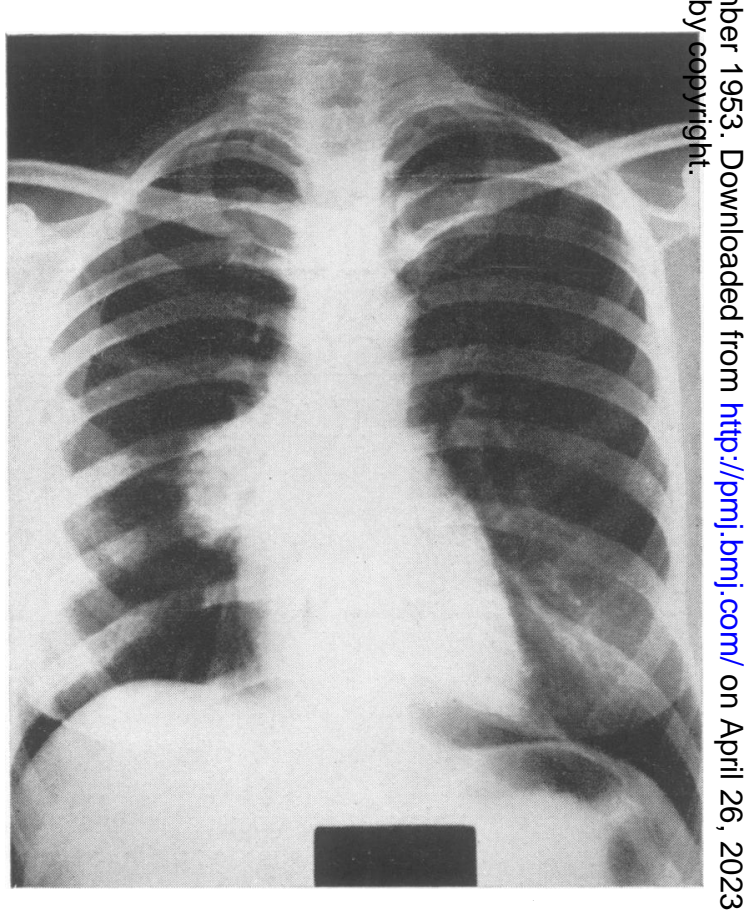

site of these symptoms will be determined by the position of the secondary deposit. Carcinoma of $\frac{\mathscr{D}}{\mathscr{D}}$ the bronchus metastasizes commonly in the spine, $\stackrel{0}{\rightarrow}$ skull, brain, liver and occasionally the skin, and 0 symptoms from any of these deposits may be the $\overrightarrow{0}$ 
first sign that something is going wrong. There are certain features which may suggest that the real lesion is a primary carcinoma, for example, with secondary deposits in the spine, pain over the site of the lesion associated with root pain are always suggestive. Secondly, tenderness of a particular vertebra to percussion is very suggestive that the body of the vertebra may be involved, and if there is evidence of destruction to the vertebra radiologically, the possibility of a primary growth in the lung should always be considered, although, of course, it may be elsewhere. In secondary deposits in the skull the history is usually helpful. The patient complains of headaches, but this is frequently a headache which is localized to one small area of the skull which may be tender to palpation and is unlike the headache which is associated with other conditions, which is usually more generalized and often at the top of the head or unilateral, without localized tenderness to palpation. Lumps occurring in the skin may be the first symptom of a carcinoma of the bronchus. A biopsy of one of the lesions will settle this, but a carcinoma of the bronchus is the most common carcinoma to metastasize to the skin and it may do so when the primary lesion is quite small. Secondary deposits occurring as the initial onset may, of course, appear anywhere. In cerebral lesions the symptoms will depend upon the position of the secondary deposits, and if situated below the tentorium will give rise to early pressure symptoms such as nausea, vomiting and papilloedema. In the presence of secondary cerebral deposits, while there may be alterations in personality and changes in mentality, this is not necessarily so. Rarely there may be a secondary deposit in the retina, giving rise to a localized diminished field of vision in one eye, and this can be seen opthalmoscopically. It should be pointed out that when the symptoms of the disease are due to secondary deposits it is too late for any radical treatment.

\section{Summary}

The unusual manifestations of carcinoma of the 3 lung are those where the main features are non- iु pulmonary. They occur by involving the veins, $\dot{\omega}$ nerves, oesophagus, pericardium and myocardium ${\underset{\infty}{\infty}}_{\infty}$ in the mediastinum, and there may be distal of lesions due to secondary deposits where the 8 primary lesion is small and gives rise to no 은 symptoms. There is another group in which acute $\rightarrow$ infections of the chest may be the mode of onset, $\mathbb{D}$ such as pneumonia or a pleural effusion and, $\overparen{\Phi}$ lastly, the unusual and unexplained symptoms of 3 polyarthritis and clubbing of the fingers and toes associated with a primary pulmonary neक्ष $\overrightarrow{0}$ plasm.

\title{
CARCINOMA OF THE BRONCHUS: RADIOLOGICAL ASPECTS
}

\author{
By G. Simon, M.D., D.M.R.E., F.F.R. \\ St. Bartholomew's Hospital, London
}

A radiological examination may yield most valuable evidence in favour of a diagnosis of carcinoma of a bronchus. On the other hand, it is essential to remember that, no matter what the type or site of such a lesion, a plain routine anterior-view radiograph may either fail to show any abnormality at all, or reveal a shadow so small that it passes undetected or is mistaken for an old healed tuberculous focus. Negative evidence derived from a single film must therefore be ignored when this complaint is suspected and must not be used as an excuse for postponing more elaborate radiological or clinical investigations, such as bronchoscopy.

The positive $\mathrm{X}$-ray appearances, which are frequently present when the patient is first examined, $\sigma$ depend largely on the site of origin of the neoplasm $N$ and, to a smaller extent, on its histological type.

The most useful division is between lesions $\sigma$ arising in the more proximal bronchi and those situated more peripherally. Of the peripheral lesions, those arising in an extreme apical site, or@ in a medial (mediastinal) site, can be separated from the rest. In addition, there are complications 0 